\title{
Variations in the course of the superior gluteal artery in relation to the lumbosacral plexus
}

\author{
By \\ Hidaka ANETAI $^{1}$, Kounosuke TOKITA
1,, Ryuhei KOJIMA
Yukio AIZAWA
, \\ ${ }^{1}$ School of Physical Therapy, Faculty of Health and Medical Care, Saitama Medical University, \\ 981 Kawakado, Moroyama-cho, Iruma-gun, Saitama 350-0496, Japan \\ ${ }^{2}$ Department of Anatomy, School of Life Dentistry at Niigata, The Nippon Dental University, \\ 1-8, Hamaura-cho, Chuo-ku, Niigata 950-8580, Japan
}

-Received for Publication, March 27, $2017-$

\begin{abstract}
Key Words: superior gluteal artery, furcal nerve, lumbosacral plexus, anatomical variation, gross anatomy
Summary: The course of the superior gluteal artery (SGA) as it passes through the lumbosacral plexus is variable. The variations of the arterial course in relation to the lumbosacral plexus have focused on statistical analysis, and it is limited arterial diversity. In this study, we investigated the positional relation between the SGA and the furcal nerve (FN): guide to segmentation of the lumbosacral plexus, arising from the L4, ie, the contribution to the femoral nerve, obturator nerve, and lumbosacral trunk. We could classify the pathway of the SGA into three types based on its positional relation to the FN. The SGA courses under the ramus from which the FN originates (Type A), under the ramus one segment below the origin of the FN (Type B), or between the obturator nerve and the lumbosacral trunk (Type C). The SGA pathway in Types A and B showed a cranial or caudal shift along with cranial or caudal deviation of the FN. In summary, the variation in the SGA pathway was correlated with cranial or caudal shift of the FN. Our findings indicate that variations of the SGA pathway are associated not only with arterial transformation, but also with diversity of the lumbosacral plexus.
\end{abstract}

\section{Introduction}

It has been described in many textbooks that the superior gluteal artery (SGA) runs on the ventral aspect of the sacral plexus and passes through the plexus to the gluteal region (Clemente, 1997; Netter, 2003; Drake et al., 2005; Standring, 2008; Agur and Dalley, 2009). Most indicate that the SGA passes between L5 and S1. This segmental level for the passage of the SGA has been corroborated in approximately $60-80 \%$ of cadavers, and many investigators have reported that the course of the SGA can be classified into several patterns (Saitou, 1925; Adachi, 1928; Kouno, 1929; Hoshiai, 1938; Shimada, 1943; Arai, 1958). However, most of these studies focused mainly on statistical analysis of the SGA path and did not examine the course of the artery in relation to peripheral nervous structures. Hoshiai (1938) examined the branching pattern of the internal iliac artery and reported the absence of any significant correlation between it and the variations of the SGA pathway. Although Kuroda et al. (2013) carefully investigated the distribution of arteries into the superior interior surface of the iliac region and suggested that it was correlated with variations in the course of the SGA, their hypothesis was limited to the arterial variations, without any reference to the relationship with nerves.

The sacral plexus is located within the pelvis, on the anterior surface of the piriformis muscle, and gives out cutaneous nerves and numerous other nerves for muscles that leave the pelvis through the great sciatic foramen. Segmental variation in the formation of the sacral plexus has been studied by various authors (Eisler, 1892; Paterson, 1894; Bardeen and Elting, 1901; Horwitz, 1939, Kawasaki, 1940; Kushida, 1940; Kudo, 1984; Chiba et al., 1994). Furthermore, a number of researchers have used the furcal nerve (FN) - the boundary between the lumbar plexus and the sacral plexus - as a guide to the arrangement of the plexus itself (Jhering, 1878; Eisler, 1892; Paterson, 1894; Bardeen and Elting, 1901; Horwitz, 1939; Kawasaki, 1940, Matsuyama, 1950; Kasai, 1957; Morikawa, 1971; Kumaki, 1981; Chiba, 1981; Kudo, 
1984; Chiba et al., 1994; Tokita et al., 2009, Anetai et al., 2010). The FN usually arises from L4 and trifurcates into branches that join the femoral nerve, obturator nerve and lumbosacral trunk. According to several reports, precise observation of the origin of the FN can explain both the remarkable variations between segments and the slight deviations within each segment (Eisler, 1892; Bardeen and Elting, 1901; Horwitz, 1939; Matsuyama, 1950; Chiba, 1981; Kudo, 1984; Chiba et al., 1994; Tokita et al., 2009, Anetai et al., 2010). Slight deviation can be judged from the size of the branch of the lumbosacral trunk originating from L4. Stated quite simply, a large size of the lumbosacral trunk arising from L4 indicates cranial deviation, while a relatively thin lumbosacral trunk arising from L4 indicates caudal deviation (Kumaki, 1981; Chiba, 1981; Kudo, 1984; Chiba et al., 1994; Tokita et al., 2009, Anetai et al., 2010). In light of these findings, we evaluated the segmental origin of the sacral plexus by considering the effect of the FN.

Although many studies have reported on the positional relation between the SGA and the sacral plexus, many have focused exclusively on the morphological variations of the artery (Saitou, 1925; Adachi, 1928; Kouno, 1929; Hoshiai, 1938; Shimada, 1943; Arai, 1958), and, as mentioned above, the relationship of variations of the SGA with variations in the segmental origin of the sacral plexus was not well analyzed in previous studies. However, it has been found that when the SGA passes between the roots of the lumbosacral plexus, its course shows some segmental transition. Therefore, the plexus should be well analyzed to investigate the diversity of the course of SGA. In the present study, we examined both the arterial variations per se and the relation between the SGA and the lumbosacral plexus.

\section{Materials and methods}

Sixteen Japanese cadavers (25 sides) used for dissection practice or for the Macro Anatomy Seminar in Niigata held at the Department of Anatomy, School of Life Dentistry at Niigata, the Nippon Dental University, Niigata (2010-2016) were examined in the present study. All cadavers were donated for use in education. This study was performed in conformity with the principles of preservation for autopsy. We dissected and carefully observed the SGA and the peripheral nervous system in the 25 lower limbs of the 16 cadavers following typical procedures.

\section{Results}

\section{Classification of the pathway of the $S G A$}

Variations of the SGA pathway were classified into four patterns on the basis of Adachi's classification. Pas- sage of the SGA between L4 and L5 (Fig. 1A), between L5 and S1 (Fig. 1B), between S1 and S2 (Fig. 1C), and between the obturator nerve and lumbosacral trunk (Fig. 1D) are shown and summarized in Fig. 2. No other pattern could be identified in the present study. In the text below, deviation in the segmental origin of the FN and the lumbosacral plexus is explained in each case.

Group 1: SGA passing between L4 and L5 (Figs. 3A, 3B and $3 C$ )

In the case illustrated in Fig. 3A, L4 trifurcates into branches joining the femoral nerve, obturator nerve and lumbosacral trunk. A few thin branches joining the lumbosacral trunk originated from L3. Therefore, the FN originates from segments $\mathrm{L} 3+\mathrm{L} 4$. In the cases illustrated in Figs. 3B and 3C, the level of origin of the FN corresponded to L4. However, in the case illustrated in Fig. 3B, the fascicle from L4 to the lumbosacral trunk was thicker than the other fascicles (large lumbosacral trunk [LLT] type). In contrast, in the case shown in Fig. 3C, the fascicle was not as thick as that shown Fig. 3B (medium lumbosacral trunk [MLT] type).

Group 2: SGA passing between L5 and S1 (Figs. 4A, 4B, $4 C$ and $4 D$ )

In the case shown in Fig. 4A, the nerves comprising the FN arose normally from L4 with normal formation (MLT type). In the cases illustrated in Fig. 4B also, the FN origin corresponded to L4, however, the fascicle from L4 to the lumbosacral trunk was thinner than the other fascicles (small lumbosacral trunk [SLT] type). In the case shown in Fig. 4C, not only L4, but also L5 trifurcates into branches joining the femoral nerve, obturator nerve and lumbosacral trunk. Therefore, the FN origin corresponded to both L4 and L5 (caudally shifted type). In the case shown in Fig. 4D, the FN origin only corresponded to L5 (caudally shifted type).

\section{Group 3: SGA passing between S1 and S2 (Fig. 5)}

In the case illustrated in Fig. 5, the roots of both L4 and L5 trifurcated into fascicles to join the femoral nerve, obturator nerve and lumbosacral trunk. The FN arose from L4 and L5 (caudally shifted type).

Group 4: SGA passing between the obturator nerve and lumbosacral trunk (Fig. 6)

In the case shown in Fig. 6, the FN origin corresponded to L4 with normal formation (MLT type).

\section{Summary of cadavers}

Figure 7 summarizes the findings from the cadavers, describing all patterns. It shows the four SGA pathways and variations in the segmental origin of the FN. As illustrated, the SGA passes between L4 and L5, between L5 and S1, between S1 and S2, or between the obturator nerve and lumbosacral trunk. The FN can originate from 

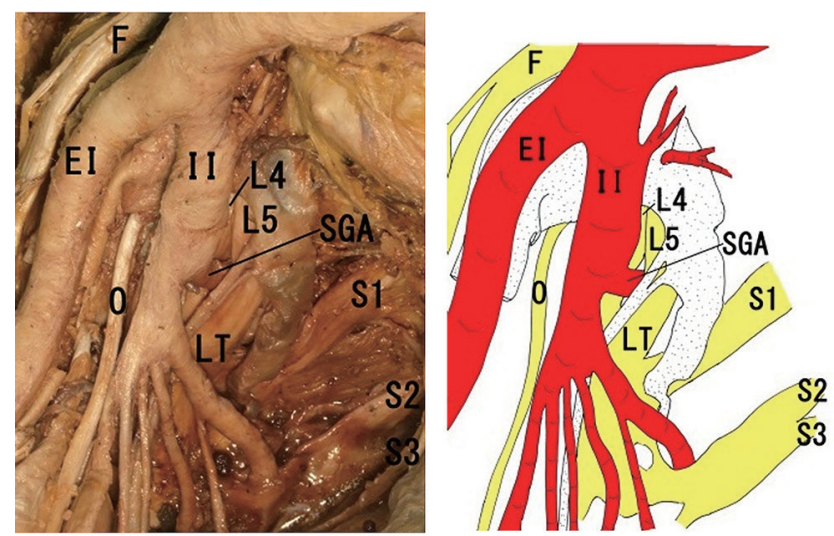

A. L4/L5
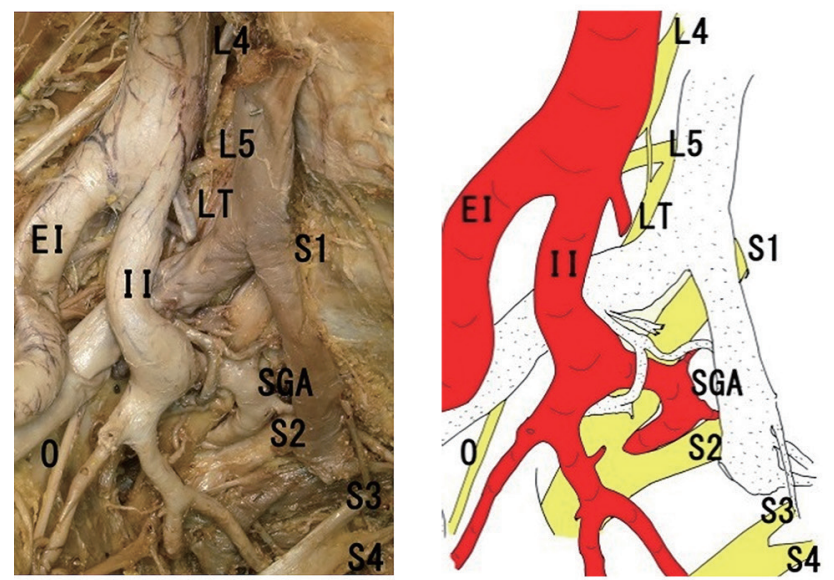

C. $\$ 1 /$ S2
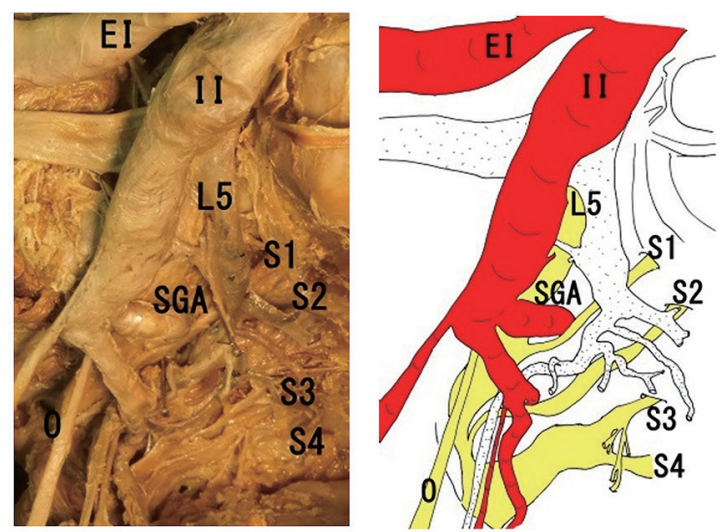

B. L5/S1
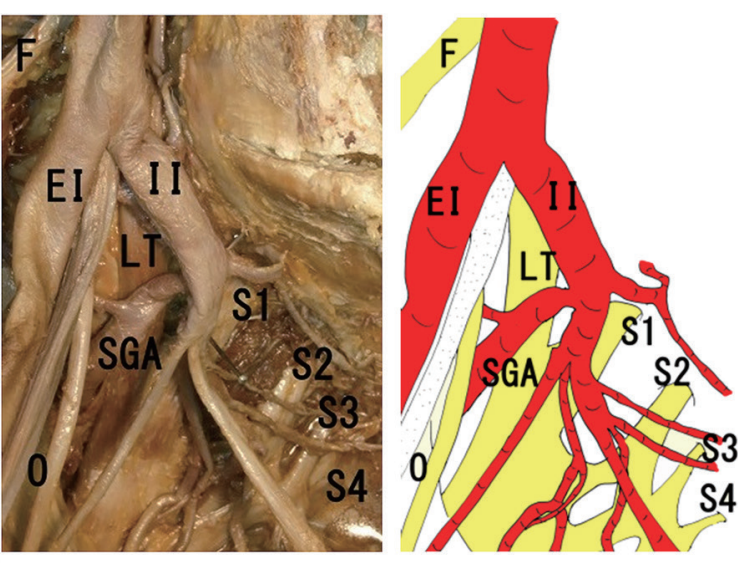

D. $0 / \mathrm{Tr}$

Fig. 1. The 4 SGA paths are described using photographs and diagrams. The SGA passed through L4/L5 (A), L5/S1 (B), S1/S2 (C) or O/LT (D). EI, external iliac artery; F, femoral nerve; II, internal iliac artery; LT, lumbosacral trunk; O, obturator nerve; SGA, superior gluteal artery.

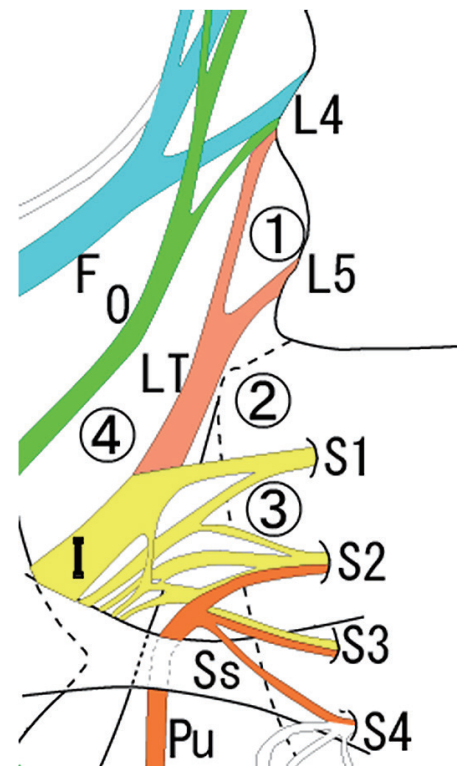

Fig. 2. Several branches arise from the lumbosacral plexus, and the SGA pathways are described using Arabic numerals. Four SGA pathways are shown. The SGA can pass between L4 and L5, between $\mathrm{L} 5$ and $\mathrm{S} 1$, between $\mathrm{S} 1$ and $\mathrm{S} 2$, or between the obturator nerve and lumbosacral trunk. F, femoral nerve; LT, lumbosacral trunk; O, obturator nerve; I, ischiadic nerve. 

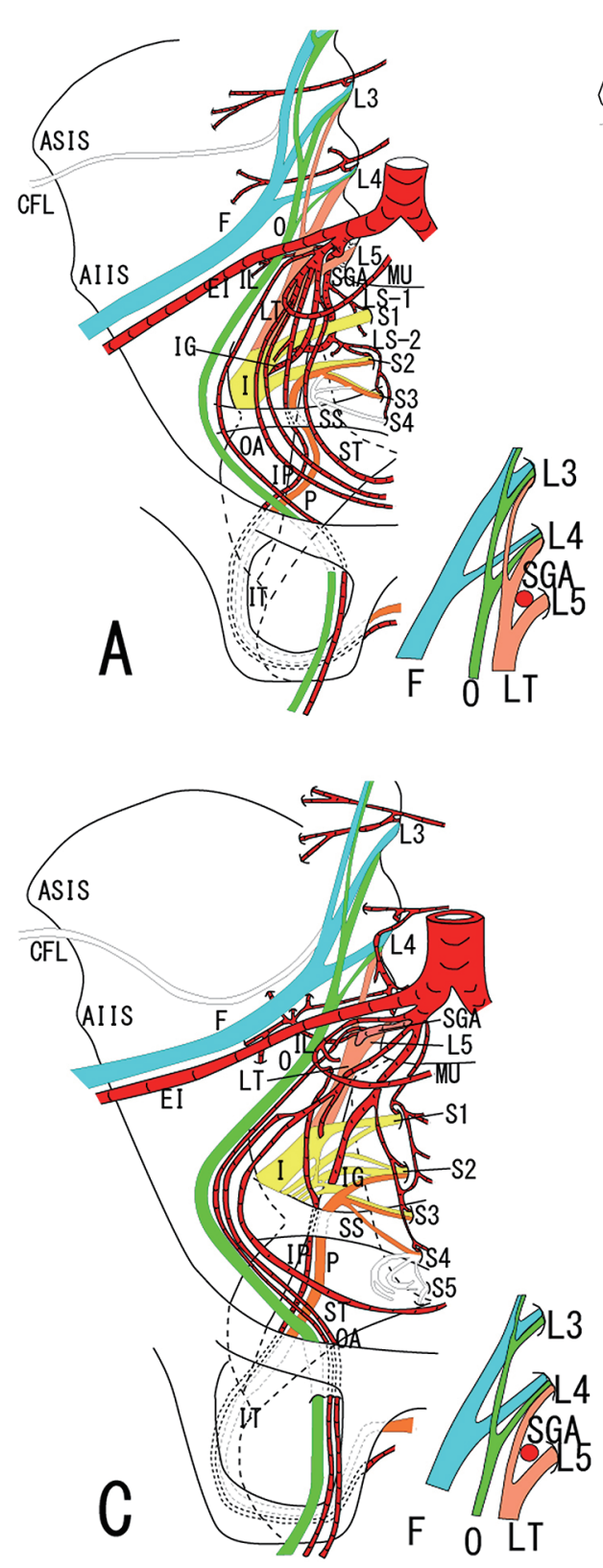

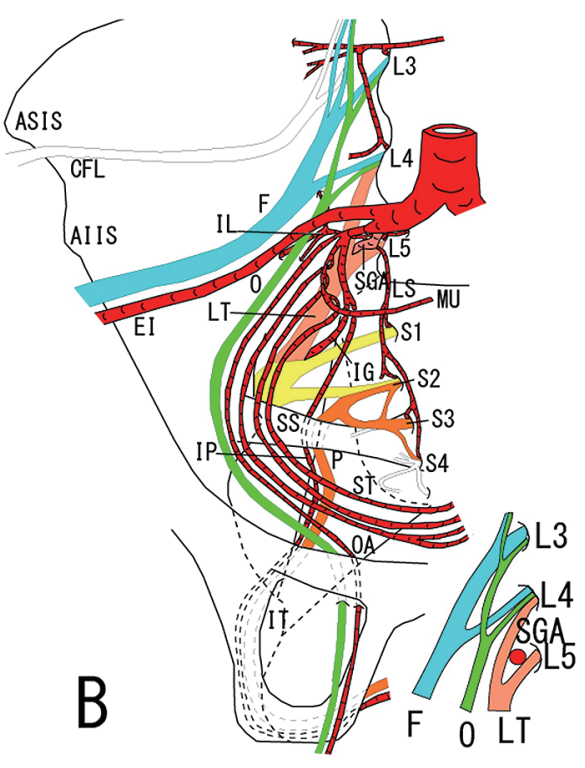

Fig. 3. Group 1: the SGA passes between L4 and L5. Schematic diagram of the left side shows numerous nerves derived from the lumbosacral plexus and various arteries branching out from the common iliac artery. The small illustration on the lower right is a simplification of the detailed drawing and shows the positional relation between the SGA course and origin of the FN. (A) The FN originates from segments L3+L4. (B) The FN originates from L4. A large branch from L4 that joins the LT. (C) The FN originates from standard segment L4. AIIS, anterior inferior iliac spine; ASIS, anterior superior iliac spine; CFL, lateral femoral cutaneous nerve; EI, external iliac artery; F, femoral nerve; I, ischiadic nerve; IG, inferior gluteal artery; IL, iliolumbar artery; IP, internal pudendal artery; IT, ischial tuberosity; LS, lateral sacral artery; LT, lumbosacral trunk; MU, medial umbilical artery; O, obturator nerve; OA, obturator artery; P, pudendal nerve; SGA, superior gluteal artery; SS, sacrospinous ligament; ST, sacrotuberous ligament.
$\mathrm{L} 3+\mathrm{L} 4, \mathrm{~L} 4, \mathrm{~L} 4+\mathrm{L} 5$, or L5. Slight variations in the thickness of fascicle originating from L4 can be classified as LLT, MLT, or SLT, on the basis of the size of the branches entering the femoral nerve, obturator nerve and lumbosacral trunk originating from L4.

\section{Discussion}

In most previous studies of variations of the SGA pathway, the variations in relation to the peripheral nervous system were not investigated. On the other hand, the positional relation between vascular and peripheral nervous structures has been investigated in morphological studies of the brachial region. A number of studies have examined variations in the pathways of the axillary artery (Singer, 1923; Adachi, 1928; De Garis and Swartley, 1928; Hukuyama, 1938; Miller, 1939; Mori, 1941; Huelke, 1959; Keen, 1961; Chiba, 1986; Satou and Takahashi, 1987; Bergman et al., 1988; Aizawa et al., 1996). Most of these examinations focused on categorization and statistical analysis. Several investigators suggested that variations in the course of the axillary artery arose from morphological variations of the artery (Müller, 1904; Chiba, 1986; Aizawa et al., 1996). However, the relation between the axillary artery and brachial plexus was also carefully evaluated by several investigators, and variations of the arterial course were precisely surveyed in macroscopi- 

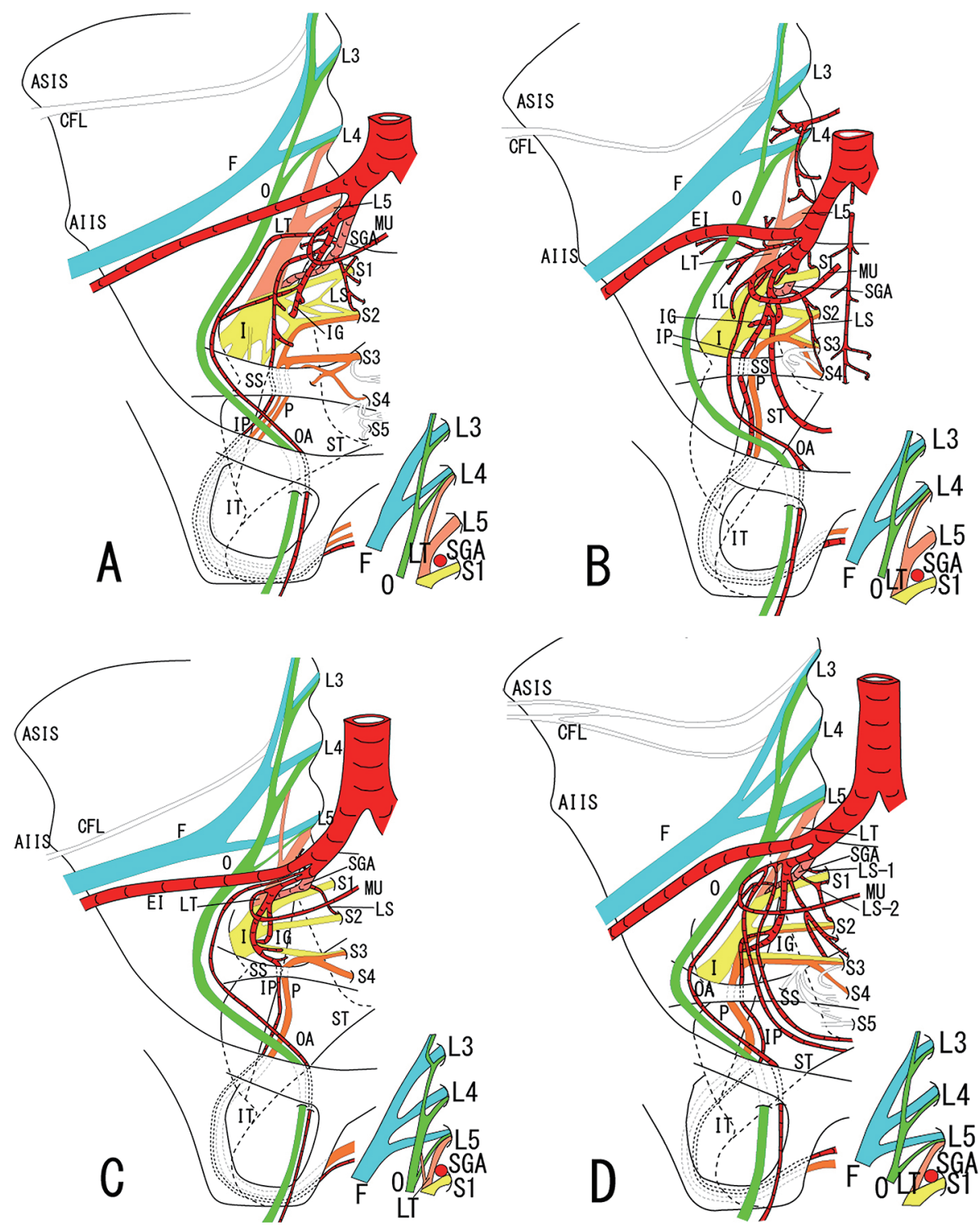

Fig. 4. Figure 4 Group 2: the SGA passes between L5 and S1. (A) The FN originates from standard segment L4. (B) The FN originates from segment L4. A small branch connecting to the LT is derived from L4. (C) The FN originates from segments L4+L5. (D) The FN originates from L5. AIIS, anterior inferior iliac spine; ASIS, anterior superior iliac spine; CFL, lateral femoral cutaneous nerve; EI, external iliac artery; F, femoral nerve; I, ischiadic nerve; IG, inferior gluteal artery; IL, iliolumbar artery; IP, internal pudendal artery; IT, ischial tuberosity; LS, lateral sacral artery; LT, lumbosacral trunk; MU, medial umbilical artery; O, obturator nerve; OA, obturator artery; P, pudendal nerve; SGA, superior gluteal artery; SS, sacrospinous ligament; ST, sacrotuberous ligament.

cally and discussed embryologically. Only a few reports have discussed the relation of the artery to the peripheral nervous system. Singer (1923) and Chiba (1984) examined the segmental composition of the brachial plexus by removing the fibrous sheath of the nerve, and Singer (1923) noted that segmental variation of the plexus did not result in variation in the course of the artery. In contrast, Chiba (1984) reported that a collateral course of the axillary artery seemed to be caused by obstruction of arterial development arising from fibrous variation of the plexus. No such arterial study of the relationship with the peripheral nervous system has been carried out for arteries of the pelvic region.

Figure 7 summarizes the results of observation of the cadavers. Figures 3-6 show the categorization based on the segments of origin the FN. The results suggest that the SGA course shifts cranially or caudally in relation to cranial or caudal deviation of the FN, although this tendency was not observed in all cases. Accordingly, the variations in the course of the SGA appeared to be correlated with 


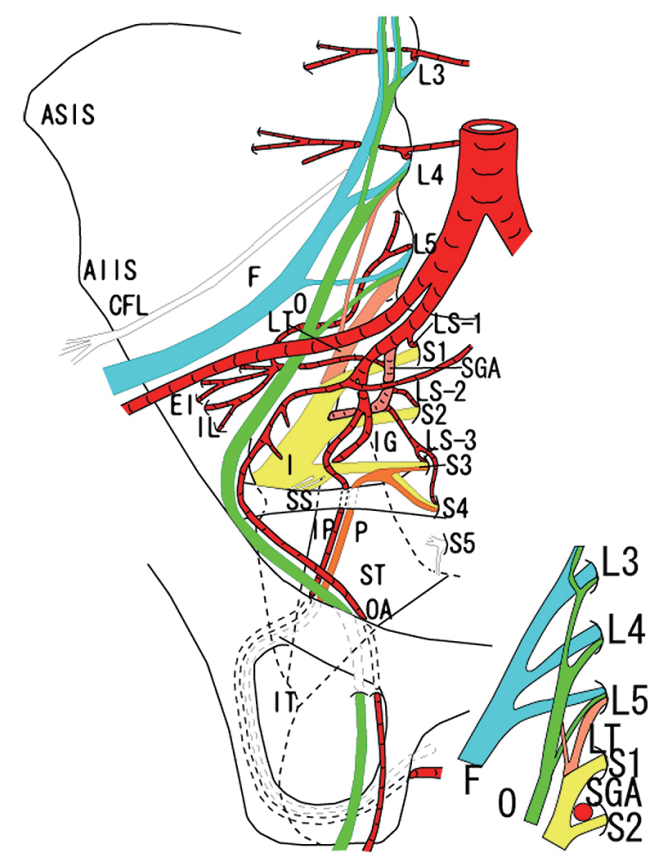

Fig. 5. Group 3: the SGA passes between S1 and S2. The FN originates from segments L4+L5. AIIS, anterior inferior iliac spine; ASIS, anterior superior iliac spine; CFL, lateral femoral cutaneous nerve; EI, external iliac artery; F, femoral nerve; I, ischiadic nerve; IG, inferior gluteal artery; IL, iliolumbar artery; IP, internal pudendal artery; IT, ischial tuberosity; LS, lateral sacral artery; LT, lumbosacral trunk; MU, medial umbilical artery; O, obturator nerve; OA, obturator artery; P, pudendal nerve; SGA, superior gluteal artery; SS, sacrospinous ligament; ST, sacrotuberous ligament.

the segmental origin of the FN and plexus. Although this is a fascinating finding, a new perspective may be necessary to describe the variations in the positional relation between the SGA and plexus, as some of the cases did not exhibit the above tendency.

\section{New categorization of the SGA pathways}

In our examination of categorical variations in the SGA, we focused on the original segments of origin of the FN. We noticed, throughout the present study, that the SGA pathway was related to the origin of the FN. Therefore, the positional relation between the pathways of the SGA and FN was evaluated. In relation to the FN, the SGA pathways were categorized into three patterns, as follows: (1) the SGA passes under the ramus from which the FN originates (Type A, Fig. 8A), (2) the SGA passes under the ramus one segment below the ramus of origin of the FN (Type B, Fig. 8B), and (3) the SGA passes between the obturator nerve and the lumbosacral trunk (Type C, Fig. 8C). In Type A, the SGA passes under the ramus from which the FN originates (Figs. 3A, 3B, 3C, 4C and 4D), between L4 and L5 (Figs. 3A, 3B and 3C) or L5 and

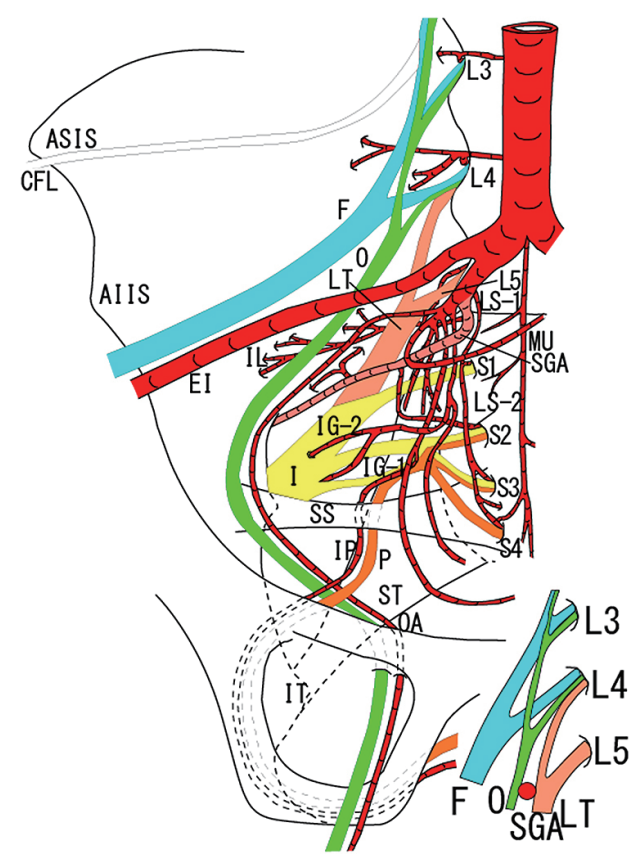

Fig. 6. Group 4: the SGA passes between the obturator nerve and lumbosacral trunk. The FN originates from L4, suggesting standard segmentation. AIIS, anterior inferior iliac spine; ASIS, anterior superior iliac spine; CFL, lateral femoral cutaneous nerve; EI, external iliac artery; F, femoral nerve; I, ischiadic nerve; IG, inferior gluteal artery; IL, iliolumbar artery; IP, internal pudendal artery; IT, ischial tuberosity; LS, lateral sacral artery; LT, lumbosacral trunk; MU, medial umbilical artery; $\mathrm{O}$, obturator nerve; OA, obturator artery; P, pudendal nerve; SGA, superior gluteal artery; SS, sacrospinous ligament; ST, sacrotuberous ligament.

S1 (Figs. 4C and D). In Type B, the SGA passes under the ramus one segment below the ramus of origin of the $\mathrm{FN}$ (Figs. 4A, 4B and 5), between L5 and S1 (Figs. 4A an 4B) or S1 and S2 (Fig. 5). In Type C, the SGA passes between the obturator nerve and the lumbosacral trunk (Fig. 6). No previous study has examined variations of the SGA based on this categorization, which revealed three distinct SGA pathways.

\section{Discussion of the new categorization scheme (Fig. 9)}

Figure 9 summarizes the three SGA pathways (Type A, $\mathrm{B}$ and $\mathrm{C}$ ), which are demarcated by the two dotted lines. Type C SGA is detailed in the left section of Fig. 9. It passes between the obturator nerve and the lumbosacral trunk, and the FN originates from standard segmentation. Type A cases are indicated in the center section of Fig. 9. When the SGA passes between L4 and L5, the FN originates from L3+L4 or L4 (LLT or MLT type, respectively), and its position suggests cranial deviation or standard segmental composition of the plexus. For SGAs coursing between L5 and S1, the segment of origin of the FN was L4+L5 or L5, and the FN was shifted caudally. The de- 

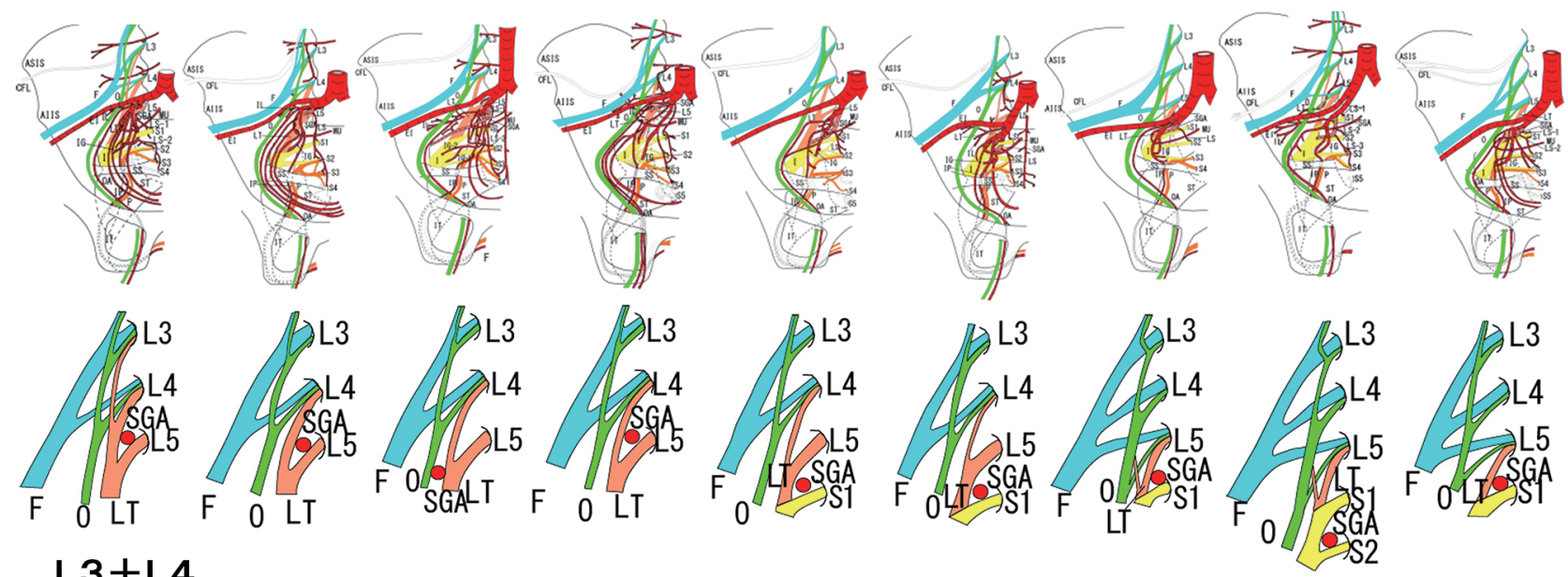

L3+L4

L4 (LLT)

L4 (MLT)

The origin of the FN

L4 (SLT)

\section{O/LT(L4/L4)}

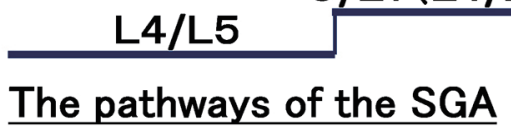

L4/L5

The pathways of the SGA

\section{S1/S2}

Fig. 7. Summary of the four SGA pathways and six segmental variations of the FN. Precise drawings of the cases are arranged by level of origin of the FN. The two lines below the illustrations indicate the level of FN and the SGA pathway. AIIS, anterior inferior iliac spine; ASIS, anterior superior iliac spine; CFL, lateral femoral cutaneous nerve; EI, external iliac artery; F, femoral nerve; I, ischiadic nerve; IG, inferior gluteal artery; IL, iliolumbar artery; IP, internal pudendal artery; IT, ischial tuberosity; LS, lateral sacral artery; LT, lumbosacral trunk; MU, medial umbilical artery; O, obturator nerve; OA, obturator artery; P, pudendal nerve; SGA, superior gluteal artery; SS, sacrospinous ligament; ST, sacrotuberous ligament.

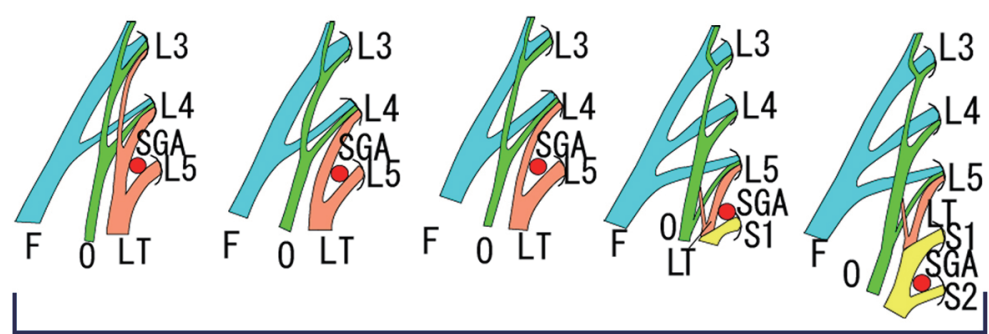

Type A
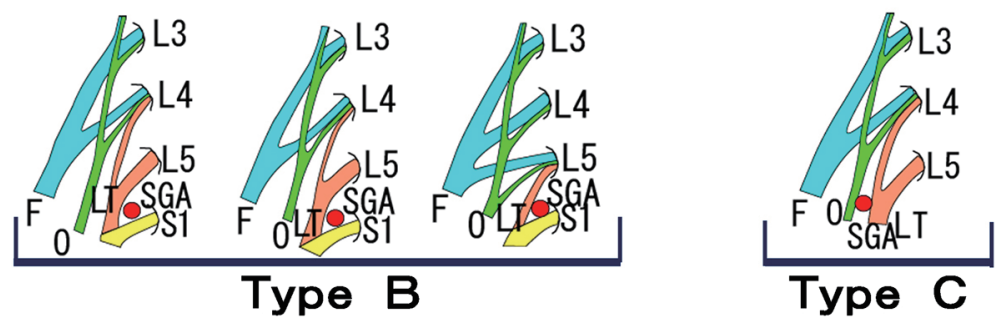

Fig. 8. New scheme for classifying variations of the SGA pathways shows the positional relation between the SGA pathways and segmentation of the FN. The SGA pathway can be classified into three types, according to its positional relation to the FN. The SGA may pass under the ramus from which the FN originates (Type A), under the ramus one segment below the origin of the FN (Type B), or between the obturator nerve and the lumbosacral trunk (Type C). F, femoral nerve; LT, lumbosacral trunk; O, obturator nerve; SGA, superior gluteal artery. 


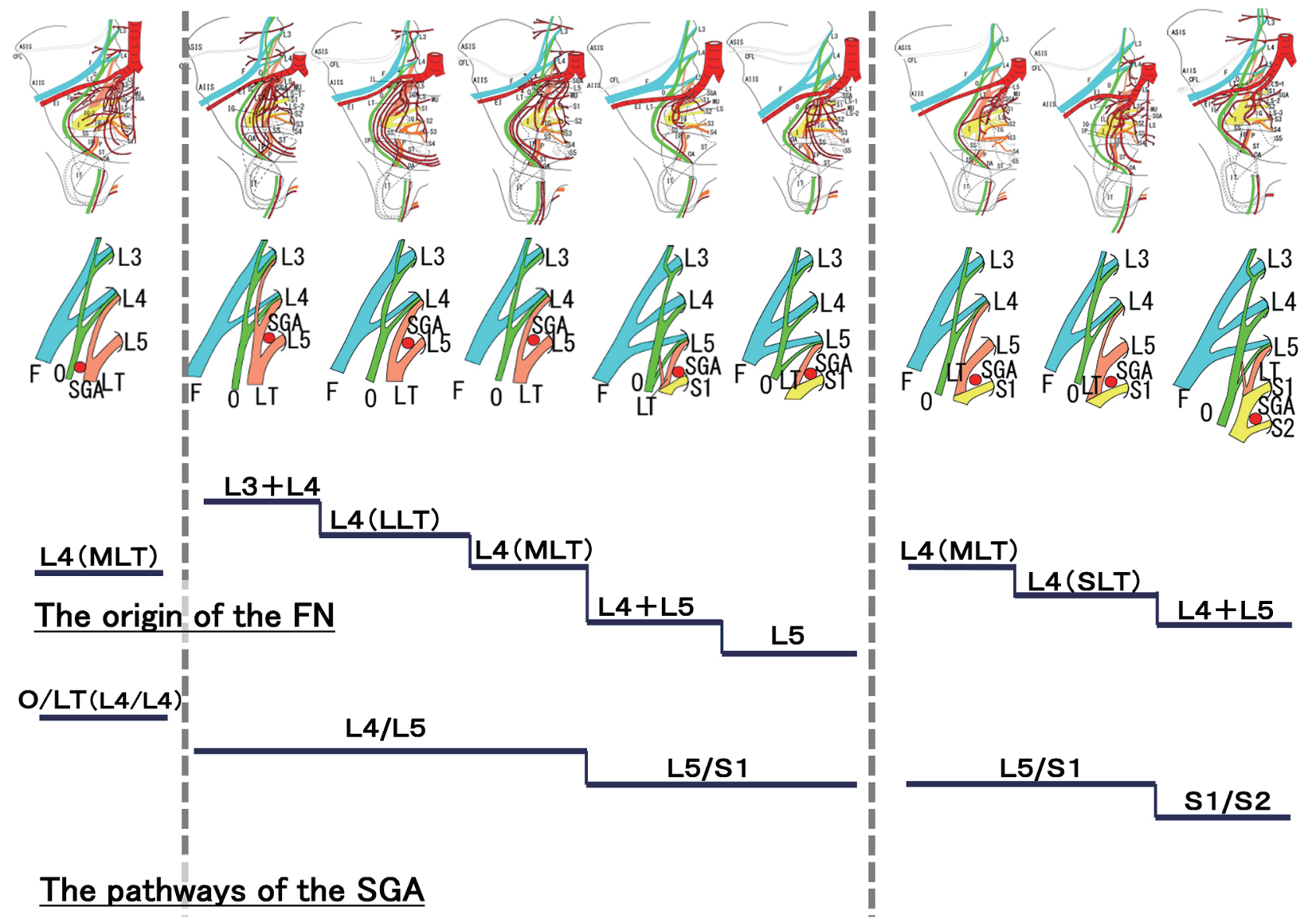

Fig. 9. Summary of the three patterns of the SGA pathways and six segmental variations of the FN. The nine illustrated cases are described according to the level of the FN. The two lines below the illustrations indicate the level of the FN and the SGA pathway with respect to each other. The two dotted lines demarcate the three patterns of the new categorization. AIIS, anterior inferior iliac spine; ASIS, anterior superior iliac spine; CFL, lateral femoral cutaneous nerve; EI, external iliac artery; F, femoral nerve; I, ischiadic nerve; IG, inferior gluteal artery; IL, iliolumbar artery; IP, internal pudendal artery; IT, ischial tuberosity; LS, lateral sacral artery; LT, lumbosacral trunk; MU, medial umbilical artery; O, obturator nerve; OA, obturator artery; P, pudendal nerve; SGA, superior gluteal artery; SS, sacrospinous ligament; ST, sacrotuberous ligament.

tails of the center section of Fig. 9 indicate that variations of the SGA pathway result in cranial or caudal deviation of the FN. Type B cases are illustrated in the right section of Fig. 9. When the SGA passes between L5 and S1, the FN originates from L4 (MLT or SLT type), and standard segmentation or slight caudal deviation is present. When the SGA passes between S1 and S2, the FN originates from segments L4+L5. Therefore, the FN and plexus exhibit caudal deviation. These cases and the Type A cases demonstrate that the SGA pathway is strongly related to the behavior of the peripheral nervous system. As mentioned above, this correlation between the SGA and FN was observed as a consequence of our new evaluation scheme, which is based on the FN. Our findings suggest the existence of a close relationship between the SGA pathway and variations in the segment of origin of the FN.

\section{Causes of anatomical variations of the $S G A$}

In addition to developing a new scheme for categorizing variation of the SGA, a three-dimensional graph (Fig. 10) was used to analyze the diversity of the positional relations between the SGA and the lumbosacral plexus. Figure 10 shows the relation between the three morphological variables, namely, course of the SGA in relation to the FN (shown on the $\mathrm{X}$ axis), segmental variation of the FN (shown on the Y axis), and the SGA pathway in the roots of the lumbosacral plexus (shown by the $\mathrm{Z}$ axis). As shown in Fig. 10, there are three ways by which the SGA passes through the plexus around the FN, and the FN can shift cranially or caudally. These differences in the course of the SGA suggest that the variations are caused by both arterial transformation and segmental deviation in the nervous system, namely, that $\mathrm{Z}$ axis variations include the $\mathrm{X}$-axis and $\mathrm{Y}$ axis variations. 


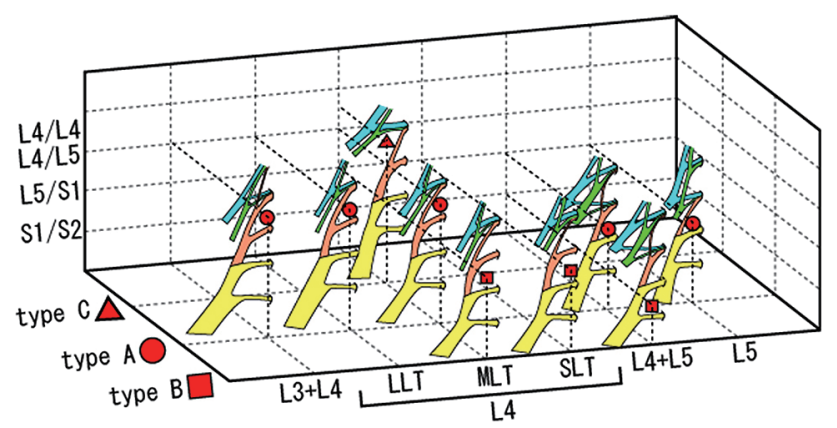

Fig. 10. Three-dimensional graph of the three SGA pathways. The three variations in the pathways of the SGA are indicated on the $\mathrm{X}$ axis, by circles, squares and triangles indicate the SGA pathways, which are classified according to the positional relation to the FN segmentation. Segmental variation of the FN is classified according to the level of segmental constitution and is shown on the $\mathrm{Y}$ axis. The SGA pathways are classified according to the traditional scheme on the $\mathrm{Z}$ axis. FN, furcal nerve; SGA, superior gluteal artery.

\section{Acknowledgments}

The authors are grateful to the cadaver donors and their families.

\section{Conflict of interest}

None.

\section{References}

Adachi B: Das Arterien system der Japaner. Bd I, Maruzen Co, Kyoto, 1928; 196-326.

Agur AM. R, Dalley AF: Grant's Atlas of Anatomy. Twelfth Edition. Lippincott Williams \& Wilkins, Philadelphia 2009; 206, 224, 242

Aizawa Y, Otsuka K, Kumaki K: Examination of the Course of the Arteries in the Axillary Region II. The Course of the Axillary Artery in the Case of Adachi's C-type Brachial Plexus. Kaibogaku Zasshi 1996; 71:92-105 (in Japanese with English abstract).

4Anetai H, Tokita K, Kojima R, Akita M, Nagashima M: The 13th rib and the lumbar plexus. Structure and Function 2010; 9:30 (in Japanese).

Arai M, Gotanda Y: Anatomical studies on the pelvic vessels of the Japanese. Kaibogaku Zasshi 1958; 33:165-166 (in Japanese).

Bardeen CR, Elting AW: A statistical study of the variations in the formation and position of the lumbosacral plexus in man. Part II. Anat Anz 1901; 19:209-232.

Bergman RA: Compendium of Human Anatomic Variation. Urban \& Schwarzenberg, Baltimore, 1988; 374-379.

Chiba S: Interrelation between the furcal nerve and the vertebral segmentation. Kaibogaku Zasshi 1981; 56:523-524 (in Japanese).

Chiba S: Morphological Studies of the So-Called Adachi's C-Type Brachial Plexus 2. Fasciculation of the Plexus and Segmental Constitution of the Nerves. Kaibogaku Zasshi 1984; 59: 707-722 (in Japanese with English abstract).

Chiba S, Ishibashi Y, Kasai T: Perforation on Dorsal Branches of the
Sacral Nerve Plexus though the Piriformis Muscle and Its Relation to Changes of Segmental Arrangements of the Vertebral Column and others. Kaibogaku Zasshi 1994; 69:280-305 (in Japanese with English abstract).

Clemente CD: Anatomy. A Regional Atlas of Human Body. Fourth Edition, Lippincott Williams \& Wilkins, Philadelphia 1997; 266.

De Garis CF, Swartley WB: The axillary artery in White and Negro stock. Am J Anat, 1928; 41:353-397.

Drake RL, Vogl AW, Mitchell AWM, Tibbitts RM, Richardson PE: Gray's Anatomy for Students. 39th Edition. Elsevier Churchill Livingstore, London, 2005; 429.

Eisler P: Der Plexus lumbosacralis des Menschen. Abh Naturforsch Ges Halle 1892; 17:280-364.

Horwitz MT: The anatomy of (A) the lumbosacral nerve plexus- its relation to variations of vertebral segmentation, and (B), the posterior sacral nerve plexus. Anat Rec 1939; 74:91-107.

Hoshiai G: Über die Beckenarterien der japanischenfeten. Kaibogaku Zasshi 1938; 11:61-72 (in Japanese with german abstract).

Huelke DF: Variation in the origins of the brachial artery. Anat Anz 1959; 135:33-41.

Hukuyama U: The study of the artery of the upper limb in northern Chinese, I, on the artery of the upper arm. Mansyu Igakkai Kaishi 1938; 29:241-269 (in Japanese).

Jhering H: Das perpherische Nerven-system der Wirbel-thiere, als Grundlage für die kenntnisse der Region enbildung der Wirbelsäule F. C. W. Vogel, Leipzig, 1878; 1-33.

Kasai T: PRI LA ABDOMENMURAJ BRANĜOJ DE LA LUMBA PLEKSO. Kaibogaku Zasshi 1957; 32:262-277 (in Japanese with Esperanto abstract).

Kawasaki Y: The study of the lumbosacral plexus in Korean. Chosen Igakkai Zasshi 1940; 30:305-331 (in Japanese).

Keen JA: A study of the arterial variations in the limbs, with special reference to symmetry of vascular patterns. Am J Anat 1961; 108: 245-261.

Kouno M: The study of the superior gluteal artery, inferior the gluteal artery and the internal pudendal artery in Japanese adults. Kaibogaku Zasshi 1929; 2:631-664 (in Japanese).

Kudo O: An anatomical study on the lumbo-sacral nerve root syndrome. Orthop Surg 1984; 35, 7:775-784 (in Japanese).

Kumaki K: Lumbar plexus as the transitory nerves of the lower abdominal wall. Kaibogaku Zasshi 1981; 56:522-523 (in Japanese).

Kuroda Y, Kudo H, Sakai T: The study of the artery distributes to the superior interior surface of pelvis. Proceeding of the 118th annual meeting of the Japanese association of anatomist 2013; 189 (in Japanese).

Kushida T: Uber die Lumbosacralnerven der japanischenfeten. II. Mitteilung (Sakralnerven). Kaibogaku Zasshi 1940; 15:486-508 (in Japanese with german abstract).

Matsuyama T: Über den plexus lumbalis bei Japanern Folia Psychiatr Neurol Jpn 1950; 4:230-286.

Mori Y: Die Arterien der oberen Extremitäten bei japanischenfeten. I. Oberarmteil. Kaibogaku Zasshi 1941; 18:179-195 (in Japanese with german abstract).

Morikawa R: Course, distribution and their variety of the nerves derived from the lumbar plexus to supply the abdominal wall. Kaibogaku Zasshi 1971; 46:312-338 (in Japanese with English abstruct).

Miller RA: Observation upon the arrangement of the axillary artery and the brachial plexus. Am J Anat 1939; 64:143-163.

Netter FH: Atlas of Human Anatomy. Third Edition. ICON Learningsystems, New York, 2003; 481.

Paterson AM: The origin and distribution of the nerve to the lower limb. J Anat Phisiol 1893/1894; 28:84-95, 169-193.

Saitou G: On the pelvis vein in the Japanese woman. Mansyu Igakkai Kaishi 1925; 3:609-677 (in Japanese).

Sato T, Takahashi T: The Axillary artery in Japanese Adults I. Kyorin Igakkai Zasshi 1987; 18:195-211 (in Japanese with English abstract). 
Shimada W: The study of the artery of the lower limb in Japanese fetus: the artery of the pelvis. Keio Igaku 1944; 24:393-417 (in Japanese).

Singer E: Human brachial plexus united into a single cord. Anat Rec 1923; 55:411-419.
Standrings: Gray's Anatomy. The Anatomical Basis of Clinical Practice. 40th Edition. Elsevier Churchill Livingstore, London, 2008; 1087.

Tokita K, Asamoto K, Nagashima M, Nakano T, Kumaki K: Morphological analysis of the lumbosacral trunk. Kaibogaku Zasshi 2009; 84:235 (in Japanese). 